\title{
Financement De La Caisse De Solidarité Du District Sanitaire De Youwarou Au Mali : Une Revue Systématique De Littérature
}

\author{
Seydou Kanté
}

Centre de Santé de Référence de Youwarou, Direction Régionale de la Santé de Mopti, Mali

Ousmane Sylla

Direction Générale de la Santé et de l’Hygiène Publique Bamako, Mali

Valeria Campos Da Silveira

Institut de Médecine Tropical (IMT) d’Anvers, Belgique

Ousmane Touré

Institut Nationale de Formation en Sciences de la Santé (INFSS) Bamako, Mali

\section{Zakaria Keita}

Consultant Indépendant en Santé - Association la Rage en Afrique de

l’Ouest, Mali

\section{Doi:10.19044/esj.2021.v17n3p190}

Submitted: 20 April 2020

Accepted: 12 January 2021

Published: 31 January 2021
Copyright 2021 Author(s)

Under Creative Commons BY-NC-ND

4.0 OPEN ACCESS

Cite As:

Kanté S., Sylla O., Campos Da Silveira V., Touré O. \& Keita Z. (2021). Financement de la Caisse de Solidarité du District Sanitaire de Youwarou au Mali : une revue systématique de littérature. European Scientific Journal, ESJ, 17(3), 190.

https://doi.org/10.19044/esj.2021.v17n3p190

\section{Résumé}

Objectif : Evaluer le mécanisme de financement de la caisse de solidarité dans le cadre du système de référence-évacuation du district sanitaire de Youwarou. Méthode : Il s’agit d'une revue systématique de la littérature réalisée du 1er Mai au 30 Juin 2017 sur le district de Youwarou. Les données sont issues de la littérature accessible sur internet et de la littérature grise (plans d'actions, documents de politique, rapports) disponible au Mali. Les moteurs de recherche PUBMED et Google scholar ont été utilisés. Les informations ont été répertoriées dans les rubriques suivantes : i) contexte, ii) processus, iii) contenu et iv) acteurs selon le modèle proposé par Gilson et 
Walt. Résultat : Le financement de la caisse de solidarité lancé en 2006 a connu d'énormes insuffisances de financement, ainsi le taux de paiement des quotes-parts de 2006 à 2009, était de $43 \%$ pour les mairies, 73\% pour les ASACO et de 15\% pour le conseil de cercle. Malgré la révision en 2013 ayant abouti au scénario de paiement par les ménages, le taux de paiement n'était que de $12 \%$. Conclusion : Le déficit de financement de la caisse de solidarité du système de référence-évacuation est lié au faible engagement et de contribution des collectivités décentralisées et des associations de santé communautaire. Un plaidoyer auprès des acteurs communautaires pour plus d'engagement de mobilisation des ressources au niveau des collectivités rurales en vue d'assurer un système de référence évacuation fiable et réduire ainsi les décès maternels et néonataux.

Mots-clés: Référence-Évacuation, Financement, Promotion De La Santé, Femmes Enceintes, Communauté Rurale, Mali

\section{Financing Of The Solidarity Fund Of The Health District Of Youwarou In Mali: A Systematic Review Of The Literature}

\section{Seydou Kanté}

Centre de Santé de Référence de Youwarou, Direction Régionale de la Santé de Mopti, Mali

Ousmane Sylla

Direction Générale de la Santé et de l’Hygiène Publique Bamako, Mali

Valeria Campos Da Silveira

Institut de Médecine Tropical (IMT) d'Anvers, Belgique

Ousmane Touré

Institut Nationale de Formation en Sciences de la Santé (INFSS) Bamako, Mali

Zakaria Keita

Consultant Indépendant en Santé - Association la Rage en Afrique de

l’Ouest, Mali

Abstract

Objective: Evaluate the financing mechanism of the solidarity fund within the framework of the referral-evacuation system of the Youwarou health district. Method: This is a systematic review of the literature carried out from May 1 to June 30, 2017 in the district of Youwarou. The data are resulted of the literature available on the internet and gray literature (action plans, policy documents, reports) available in Mali. The PUBMED and 
Google scholar search engines were used. The information has been listed under the following headings: i) context, ii) process, iii) content and iv) actors according to the model proposed by Gilson and Walt. Result: The financing of the solidarity fund launched in 2006 experienced enormous funding shortfalls, so the rate of payment of quotas from 2006 to 2009 was $43 \%$ for municipalities, $73 \%$ for ASACOs and $15 \%$ for the circle council. Despite the revision in 2013 that resulted in the household payment scenario, the payment rate was only $12 \%$. Conclusion: The financing deficit of the solidarity fund of the referral-evacuation system is linked to the weak commitment and contribution of decentralized communities and community health associations. Advocacy with community actors for more commitment to mobilize resources at the level of rural communities in order to ensure a reliable referral-evacuation system and thus reduce maternal and neonatal deaths.

Keywords: Reference-Evacuation; Financing; Health Promotion; Pregnant Women; Rural Community; Mali

\section{Introduction}

Dans le monde, environ 830 femmes meurent chaque jour à cause des complications liées à la grossesse ou à l'accouchement. En 2015, 303000 femmes sont décédées pendant la grossesse ou à la suite de l'accouchement (1). La majeure partie de ces décès se sont produits dans des pays à revenu faible et la plupart auraient pu être évités. Ces centaines de milliers de femmes qui meurent en donnant la vie au $21^{\text {ème }}$ siècle est inacceptable. En Afrique subsaharienne, un certain nombre de pays ont réduit de moitié le taux de mortalité maternelle depuis 1990 (1).

Pour réduire le taux de mortalité maternelle, estimé à 577 pour 100000 naissances vivantes (2), le gouvernement du Mali a mis en place un système de référence-évacuation (SRE) pour les-urgences obstétricales, qu'on définit comme étant un état grave lié à la grossesse ou à l'accouchement et qui requiert une intervention médicale d'urgence afin d'empêcher la mort probable de la femme (3). Ce système de référence vise à améliorer la qualité des soins obstétricaux d'urgence, mais aussi l'accès des femmes à ces soins, et cela dans le but de diminuer la mortalité liée aux complications obstétricales. Brièvement, il est articulé autour de trois composantes principales : un système de financement (des caisses de solidarité) ; un système d'alerte et d'évacuation sanitaire (des ambulances et des radios) ; la mise à jour des soins obstétricaux d'urgence dans les Centres de Santé Communautaire (CSCom) et les Centres de Santé de Référence (CSRéf) $(4,5)$. 
Quant aux caisses de solidarité, elles ont été mises en place dans chaque district pour réduire les coûts assumés par les femmes lors d'une complication. Des partenaires locaux (ASACO, Mairies, Conseils de cercle, CSRéf) font des contributions financières à la caisse sur une base régulière ; la contribution de chaque partenaire étant définie annuellement par consensus. L'argent de la caisse collecté par un comité de gestion est utilisé en cas d'évacuation, pour payer le carburant de l'ambulance, le perdiem du chauffeur et de l'agent de santé qui accompagne l'ambulance, mais aussi de l'amortissement de l'ambulance et les soins fournis. Avant la mise en place des caisses de solidarité, la totalité de ces coûts devait être assumée par les patientes, ce qui constituait une barrière financière importante et pouvait retarder de façon considérable l'obtention de soins appropriés $(4,5)$.

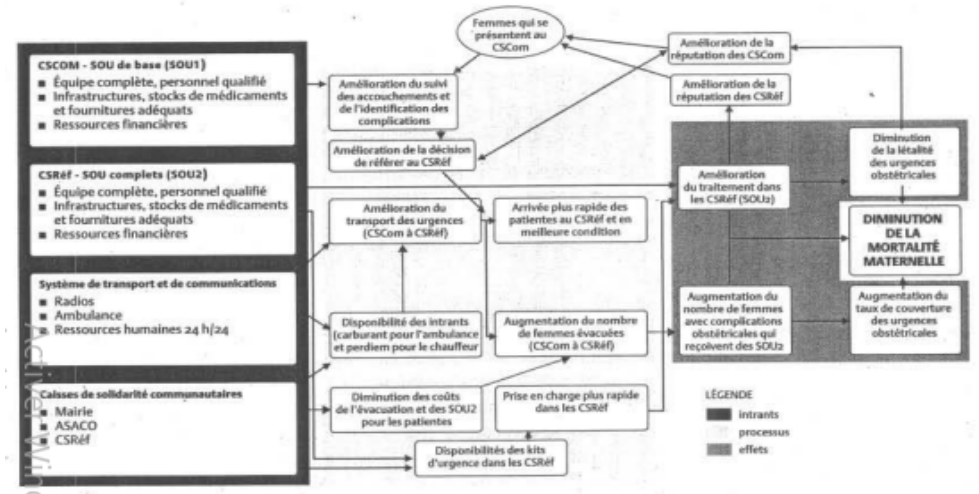

Figure 1.Cadre conceptuel du système de reference-évacuation au Mali

Dans le district sanitaire de Youwarou, les acteurs locaux, qui sont les Associations de Santé Communautaire (ASACO) et les Collectivités décentralisées (communes, conseil de cercle) sont responsable du transport des patientes des CSCom vers le CSRéf $(6,7)$. Rappelons à titre d'exemple que dans ce district sanitaire, le coût d'une seule évacuation d'un CSCom vers le CSRéf peut parfois atteindre 102.000 FCFA (environ $160 €$ ) en fonction de la période de crue ou de décrue. De ce fait, les parturientes en difficultés d'accouchement ou leurs parents supportent difficilement les frais de ces évacuations $(6,7,8)$.

L'objectif de ce travail est d'évaluer le mécanisme de financement de la caisse de solidarité afin de dégager les forces et les faiblesses de ce système mais aussi d'analyser la contribution des ménages.

\section{Methodologie}

\section{Type et site d'étude}

Il s'agit d'une revue systématique de la littérature réalisée du $1^{\mathrm{er}}$ Mai au 30 Juin 2017 sur le district de Youwarou. 


\section{Sources de données}

Elles se composent de la littérature accessible sur internet et de la littérature grise (plans d'actions, documents de politique, rapports) disponible au Mali.

\section{Technique de collecte des données}

En ce qui concerne la recherche sur internet, les moteurs de recherche PUBMED (pour l'anglais) et Google scholar (pour le français) ont été utilisés. Pour PUBMED, les mots clés introduits ont été : i) [transfer] and [pregnant women] and [fee] and [case management] and [Mali] and [youwarou] ; Pour Google scholar les mots clés ont été les suivants : i) [évacuation] and [référence] and [femme enceinte] and [caisse] and [solidarité] and [Mali] and [youwarou].

Les références d'autres articles ont été retenues à partir des résultats de la première recherche.

La littérature grise au Mali a été répertoriée dans les sites potentiels ministère de la santé, bureau de l'OMS au Mali, faculté de médecine et d'odontostomatologie, centres de recherche en santé, etc.) et exploitée.

\section{Organisation des données}

Les informations ont été répertoriées dans les rubriques suivantes : i) contexte, ii) processus, iii) contenu et les iv) acteurs, proposé par Gilson et Walt (1994) $(8,9,10)$.

\section{Traitement et analyse des données}

Les données ont fait l'objet d'une saisie sur le logiciel Word et d'une analyse afin de dégager les forces et les faiblesses du mécanisme de financement.

Cadre de l'étude : Le cercle de Youwarou est une collectivité territoriale du Mali dans la région de Mopti. Il compte 7 communes : Bembéré Tama, Déboye, Dirma, Dongo, Farimaké, N'Dodjiga et Youwarou.

Youwarou est un district instable sur le plan sécuritaire avec des attaques récurrentes des bandits suite aux nombreuses crises de la rébellion armée. Il arrive parfois que tous les prestataires qualifiés se retirent de la zone suite à la recrudescence des violences. Ce retrait intermittent des prestataires a un énorme impact sur les résultats de santé dans cette localité (12). Le district sanitaire a perdu beaucoup de biens (médicaments, véhicules, vandalisme des structures sanitaires) lors des rébellions de 1991 et de 2012. L'insécurité grandissante freine surtout tout élan du développement économique exposant 
généralement la population à des multiples razzias conduisant parfois à des pertes en vie humaine.

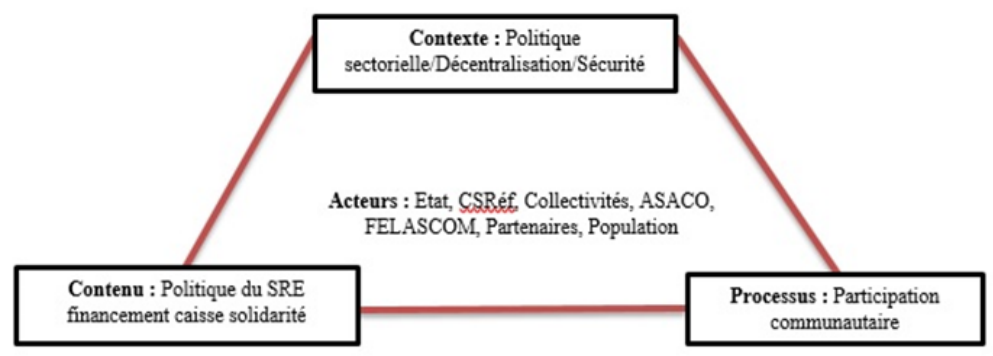

Figure 2. Cadre analytique du système de référence-évacuation du district de Youwarou (Adapté de Gilson et Walt, 1994).

\section{Resultats}

\section{Le contexte}

Le système de référence-évacuation des femmes enceintes a été mise en place au milieu des années 1990. Pour harmoniser les interventions en mai 2000, le « cadre conceptuel de l'organisation du système de référence/évacuation au Mali » a été élaboré. Ce cadre, inspiré du document sur la périnatalité du Ministère de la Santé Malien $(4,13,14,15)$, prenait en compte les aspects organisationnels, techniques, logistiques, financiers et de communication pour améliorer l'accès aux soins des populations démunies. Le système consistait à l'organisation des acteurs, à savoir, 1) l'Etat à travers les Centres de Santé de Référence (CSRéf), 2) les Associations de Santé Communautaire (ASACO), 3) les Collectivités décentralisées (communes, conseil de cercle) et 4) les parturientes et leurs parents, en vue d'assurer le transport de la parturiente référée ou évacuée depuis le CSCom jusqu'au CSRéf ainsi que sa prise en charge pour la césarienne.

Cette organisation passait par le bon fonctionnement d'une caisse de solidarité alimentée par les paiements des quotes-parts des différents acteurs ci-dessus cités. Les quotes-parts étaient reçues par le comité de recouvrement issu lui-même du comité de gestion du système de referenceevacuation. Les kits de césarienne et les actes de laboratoire pour les femmes à jour de leurs cotisations envers leur ASACO qui était elle-même à jour de ses cotisations envers la caisse de solidarité dans sa conception initiale $(4,8,13)$.

Avec l'avènement de la césarienne gratuite en 2005, la parturiente et ses parents ont été en principe dispensés du paiement des quotes-parts mais aussi des kits de césarienne. La caisse de solidarité devrait désormais être alimentée par les quotes-parts des ASACO et des collectivités territoriales (communes, conseil de cercle) qui devraient financer le transport des femmes enceintes en difficultés dans les CSCom vers les CSRéf. Les kits de 
césarienne, l'acte chirurgical et de laboratoire seraient financés par l'Etat, via un système de pré positionnement des kits et le remboursement des actes opératoires après justification des dotations antérieures (4,8,13). La Figure 2 retrace les principales réformes politiques clés en relation avec les soins de femmes enceintes.

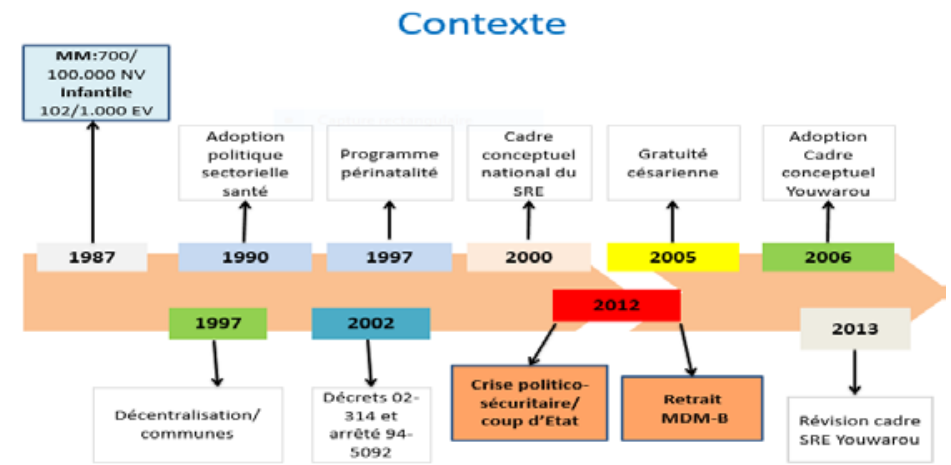

Figure 3. Evolution des réformes politiques en faveur des soins de la femme enceinte.

\section{Le contenu}

En vue de respecter la politique nationale, le système de référence/évacuation du district sanitaire de Youwarou a été mis en route en Mai 2006. Le cadre conceptuel de ce système s'est donc inspiré des cadres des autres districts sanitaires de la région de Mopti mais surtout d'autres cadres à travers le pays, en essayant de les améliorer. Différentes étapes ont été réalisées pour la mise en marche de ce système, à savoir : l'explication du mécanisme dans tous les villages du district sanitaire ; deux ateliers de consensus avec les acteurs locaux et un atelier de validation finale du cadre conceptuel $(5,6,14,15)$.

\section{Le processus}

A partir de 2005, la caisse de solidarité devrait désormais être alimentée par les quotes-parts des ASACO et des collectivités territoriales (communes, conseil de cercle) afin de financer le transport des femmes enceintes en difficultés des CSCom vers les CSRéf.

Ainsi, le seul problème qui entraverait la bonne mise en œuvre de cette politique de référence-évacuation au niveau district, pour la bonne prise en charge des femmes enceintes serait le faible taux de recouvrement des quotesparts des communes et des ASACO (16). La situation en 2006 est présentée au Tableau 1.

Tableau 1 : Répartition de la contribution des quotes-parts des acteurs

\begin{tabular}{ccc}
\hline Acteurs & Proportion \% & Contribution totale par acteurs \\
\hline Conseil de cercle (CC) & 1 & 767253 \\
ASACO & 5 & 383626 \\
Mairie & 39 & 2992287 \\
\hline
\end{tabular}




\begin{tabular}{ccc}
\hline CSRéf & 3 & 230176 \\
Etat & 52 & 3989715 \\
Total & $\mathbf{1 0 0}$ & $\mathbf{7 6 7 2 5 3 0}$ \\
\hline
\end{tabular}

Ce système a connu des difficultés de trésorerie à cause du nonpaiement des quotes-parts des acteurs locaux (les ASACO et les collectivités locales) déjà deux ans après son lancement, mettant en mal le fonctionnement de la caisse de solidarité. La caisse, qui devait couvrir à cent pour cent les frais de transport des parturientes, ne répondait donc plus qu'au cas par cas en fonction de la provenance de la patiente, contribuant à un certain refroidissement de la notion de solidarité.

Cependant, l'engouement suscité auprès de la population pendant la $1^{\text {ère }}$ année d'implantation ne cessait de croître. La population n'ayant pas connaissance que leurs responsables n'avaient pas contribué à la hauteur souhaitée continuait à solliciter l'ambulance ou la pinasse en cas de difficulté d'accouchement. Déjà aux premiers mois de l'année 2009, le CSRéf de Youwarou, émetteur des bons de carburant, s'est vu endetté auprès du fournisseur à hauteur de 900.000 FCFA (environ $1374 €$ ) pour le transport des parturientes. Cette situation a d'ailleurs été rapportée dans le rapport final d'évaluation de la césarienne au Mali (15). Ce rapport met en exergue les conséquences néfastes sur le fonctionnement courant des structures sanitaires et l'exploration des familles des parturientes obligées à contribuer aux frais de transport, créant leur désarroi. Cette contribution est cependant indispensable afin de pouvoir régler les factures des fournisseurs. L'un des problèmes entravant cette politique serait dans cette zone du Mali le faible taux de recouvrement, voire le non-paiement intégral, des quotes-parts des communes et des ASACO pour la caisse de solidarité $(11,16,17)$.

Le bon fonctionnement de la caisse de solidarité diminuerait les contraintes/barrières financières pour le transport, facilitant l'accès aux soins de santé de nombreuses femmes rurales. La situation devrait interpeller à plus d'un titre les élus locaux car les familles, malgré le payement des impôts tels les taxes du développement régional et local (TDRL), n'ont toujours pas accès aux soins.

Les collectivités locales sont restées inactives face à cette situation qui devenait de plus en plus intenable pour l'équipe technique du CSRéf. Face à cette situation, un premier monitorage sur le système de référence/évacuation avec l'ensemble des acteurs fût planifié par le CSRéf (12). L'extrait suivant de l'étude révèle ceci : "les systèmes de référence sont faciles à concevoir, mais extrêmement difficiles à mettre en pratique. L'efficacité d'un système de référence dépendra des patients, la confiance dans les différents niveaux du système de santé, la confiance qu'ils ont dans le personnel, l'efficacité du système d'information sur la facilité ou la difficulté 
de transport et le temps passé à voyager, les coûts des soins à différents niveaux » (12).

A l'issue de ce premier monitorage, le partenaire Médecins du Monde Belgique (MdM-B) a appuyé la caisse de solidarité à hauteur de cinq millions de francs CFA (environ $7634 €$ ). Malgré cet appui très important, les acteurs locaux n'avaient toujours pas pris conscience de l'ampleur du problème. Alors, en 2013, le CSRéf a procédé avec l'ensemble des acteurs à la révision du mécanisme de paiement des quotes-parts pour lui donner un nouveau souffle, ce qui a abouti à la décision du partage de coûts par ménages, via l’ASACO. Ce scenario est présenté dans le Tableau 2 (17).

Tableau 2 : Partage des coûts des quotes-parts entre les ménages

\begin{tabular}{ccccc}
\hline $\begin{array}{c}\text { Aires } \\
\text { sanitaires }\end{array}$ & $\begin{array}{c}\text { Population } \\
\text { en 2013 }\end{array}$ & $\begin{array}{c}\text { Nombre de } \\
\text { ménages }\end{array}$ & $\begin{array}{c}\text { Quote-part } \\
\text { par ménage }\end{array}$ & $\begin{array}{c}\text { Montant /aire de } \\
\text { santé }\end{array}$ \\
\hline Ambiri & 10365 & 1481 & 435 & 644235 \\
Dogo & 9182 & 1312 & 435 & 570720 \\
Gathi & 9968 & 1424 & 435 & 619440 \\
Guidio & 14574 & 2082 & 435 & 905670 \\
Kormou & 18080 & 2583 & 435 & 1123605 \\
Faou & 10731 & 1533 & 435 & 666855 \\
Sah & 18265 & 2609 & 435 & 1134915 \\
Central & 32303 & 4615 & 435 & 2007525 \\
Total & $\mathbf{1 2 3 4 6 8}$ & $\mathbf{1 7 6 3 9}$ & $\mathbf{4 3 5}$ & $\mathbf{7 6 7 2 5 3 0}$ \\
\hline
\end{tabular}

Evaluation de la contribution financière des différents les acteurs

Le Tableau 3 montre la contribution des différents acteurs sur le financement de la caisse de solidarité du système de référence/évacuation de Youwarou.

Tableau 3 : Situation de recouvrement des coûts par partenaire (2006-2009)

\begin{tabular}{ccccccc}
\hline Partenaires & Mairie & ASACO & $\begin{array}{c}\text { Conseil } \\
\text { de } \\
\text { Cercle }\end{array}$ & CSRéf & État & $\begin{array}{c}\text { Montant } \\
\text { total }\end{array}$ \\
\hline Prévu & 6264 & 954029 & 1537 & 1012 & 10304 & 20072 \\
& 072 & & 317 & 500 & 112 & 030 \\
Recouvert & 2674 & 692828 & 225000 & 810000 & 10304 & 14706 \\
$\begin{array}{c}\text { Taux } \\
\text { recouvrement }\end{array}$ & $\mathbf{4 3}$ & $\mathbf{7 3}$ & $\mathbf{1 5}$ & $\mathbf{8 0}$ & $\mathbf{1 0 0}$ & $\mathbf{7 3 \%}$ \\
$\mathbf{( \% )}$ & & & & & & \\
\hline
\end{tabular}

Il permet de constater que le financement de la caisse est resté insuffisant depuis son lancement jusqu'à nos jours avec environ $43 \%$ de taux de recouvrement.

\section{Le remboursement par les Mairies}

$\mathrm{Au}$ démarrage du système, ce sont les élus locaux (Maires) et les ASACO qui devaient mobiliser $100 \%$ du transport des parturientes. Ces 
montants n’ont jamais été recouverts à hauteur de 50\% auprès des Mairies. Le Tableau 4 renseigne qu'en dehors des paiements (42\%) de toutes les communes, réalisées pendant la période du lancement du système en Mai 2006, il n’y a plus eu de contribution de la part des communes durant les années suivantes. D’ailleurs, seule une commune a contribué à la caisse de solidarité en 2007, et les années suivantes les paiements réalisés étaient largement insuffisants. On remarque ainsi que le taux de recouvrement est resté inférieur à 50\% du budget prévu. Nombreuses études démontrent le faible accompagnement et implication des élus locaux pour les problèmes de santé de leur population $(17,18,19,20)$.

Tableau 4 : Situation de recouvrement par Mairie, District de Youwarou (2006-2009)

\begin{tabular}{|c|c|c|c|c|c|c|c|c|}
\hline \multicolumn{9}{|c|}{ Montants en FCFA } \\
\hline Années & \multicolumn{2}{|c|}{2006} & \multicolumn{2}{|c|}{2007} & \multicolumn{2}{|c|}{2008} & \multicolumn{2}{|c|}{2009} \\
\hline Mairies & $\mathbf{P}$ & $\mathbf{R}$ & $\mathbf{P}$ & $\mathbf{R}$ & $\mathbf{P}$ & $\mathbf{R}$ & $\mathbf{P}$ & $\mathbf{R}$ \\
\hline Bimbéré & 540000 & 100000 & 540000 & 0 & 540000 & 12000 & 540000 & 0 \\
\hline Deboye & 540000 & 100000 & 540000 & 540000 & 540000 & 0 & 540000 & 0 \\
\hline Dongo & 540000 & 100000 & 540000 & 0 & 540000 & 0 & 540000 & 5000 \\
\hline Farimaké & 540000 & 100000 & 540000 & 0 & 540000 & 135000 & 540000 & 100000 \\
\hline N’dodjiga & 540000 & 100000 & 540000 & 0 & 540000 & 0 & 540000 & 250000 \\
\hline Dirma & 540000 & 100000 & 540000 & 0 & 540000 & 100000 & 540000 & 100000 \\
\hline Youwarou & 540000 & 100000 & 540000 & 0 & 540000 & 270000 & 540000 & 0 \\
\hline TOTAL & 3780000 & 1575000 & 3780000 & 540000 & 3780000 & 630000 & 3780000 & 500000 \\
\hline $\begin{array}{c}\text { Contribution } \\
\text { (\%) }\end{array}$ & \multicolumn{2}{|c|}{42} & \multicolumn{2}{|c|}{14} & \multicolumn{2}{|c|}{17} & \multicolumn{2}{|c|}{13} \\
\hline
\end{tabular}

$\mathbf{P}=$ Prévu $\mathbf{R}=$ Réalisé

\section{Le remboursement par les ASACO}

Les ASACO mettaient en exergue l'insuffisance des ressources liée à la sous fréquentation des structures qui vivent du recouvrement des coûts. Le paiement des ASACO reflète presque la même tendance (43\%) que celle des communes (voir Tableau 5), laissant percevoir le faible engagement de ces associations, premières responsables des CSCom.

Tableau 5 : Situation du recouvrement par les ASACO, district de Youwarou (2006-2009)

\begin{tabular}{|c|c|c|c|c|c|c|c|c|}
\hline \multicolumn{9}{|c|}{ Montants en FCFA } \\
\hline Années & \multicolumn{2}{|c|}{2006} & \multicolumn{2}{|c|}{2007} & \multicolumn{2}{|c|}{2008} & \multicolumn{2}{|c|}{2009} \\
\hline ASACO & $\mathrm{P}$ & $\mathrm{R}$ & $\mathrm{P}$ & $\mathrm{R}$ & $\mathrm{P}$ & $\mathrm{R}$ & $\mathrm{P}$ & $\mathrm{R}$ \\
\hline Bimbéré & 64000 & 0 & 64000 & 0 & 64000 & 0 & 64000 & 0 \\
\hline Deboye & 64000 & 64000 & 64000 & 0 & 64000 & 0 & 64000 & 0 \\
\hline Dongo & 64000 & 0 & 64000 & 0 & 64000 & 0 & 64000 & 0 \\
\hline Farimaké & 64000 & 0 & 64000 & 0 & 64000 & 0 & 64000 & 0 \\
\hline N’dodjiga & 64000 & 0 & 64000 & 0 & 64000 & 0 & 64000 & 0 \\
\hline Dirma & 64000 & 64000 & 64000 & 0 & 64000 & 0 & 64000 & 0 \\
\hline Youwarou & 64000 & 64000 & 64000 & 0 & 64000 & 0 & 64000 & 64276 \\
\hline TOTAL & 450000 & 192000 & 450000 & 0 & $\begin{array}{l}450 \\
000\end{array}$ & 0 & 450000 & 64276 \\
\hline
\end{tabular}

Contribution \% 


\section{Le remboursement par ménages}

Malgré la révision du mécanisme de financement de la caisse de solidarité, le taux de recouvrement ne s’est pas amélioré. En effet, malgré l'adoption du scenario de financement de la caisse par les ménages (voir Tableau 6) via les ASACO, qui s’est montré comme une des pistes efficaces au financement de la caisse de solidarité (7). Les élus communaux, pour des fins électoralistes, ont menacé les ASACO de percevoir de l'argent sans leur intermédiation. De facto, les paiements n’ont pas atteint les seuils prévus dans aucune des aires sanitaires du district conformément au tableau 6.

Tableau 6 : Recouvrement à travers les ménages par commune, Youwarou, 2015

\begin{tabular}{cccc}
\hline $\begin{array}{c}\text { Aires de } \\
\text { santé }\end{array}$ & $\begin{array}{c}\text { Montants prévus (F } \\
\text { CFA) }\end{array}$ & $\begin{array}{c}\text { Montants recouverts (F } \\
\text { CFA) }\end{array}$ & $\begin{array}{c}\text { Taux de } \\
\text { recouvrement } \\
\text { (\%) }\end{array}$ \\
\hline Ambiri & 2576940 & 360000 & 14 \\
Dogo & 2282880 & 145000 & 6 \\
Gathi & 2477760 & 490000 & 20 \\
Guidio & 3622680 & 500000 & 14 \\
Faou & NA & NA & NA \\
Kormou & 4494420 & 786350 & 17 \\
Sah & 7207080 & 825995 & 11 \\
Y. Central & 8030100 & 698445 & 9 \\
Total & $\mathbf{3 0 6 9 1 8 6 0}$ & $\mathbf{3 8 0 6} \mathbf{1 9 0}$ & $\mathbf{1 2}$ \\
MdM/F & 4000000 & 2000000 & 50 \\
\hline
\end{tabular}

\section{Les forces et les faiblesses du système de financement de la caisse de solidarité du district sanitaire de Youwarou} Les forces

Le système de référence-évacuation élaboré est un complément pour renforcer le système des SSP. Il tire sa force et sa légitimité dans le processus de sa mise en œuvre : (i) par la réalisation des approches village afin d'expliquer de long en large les objectifs et le but de la politique à la population ; (ii) son adoption à l'unanimité en présence du représentant de l'Etat (Préfet) à l'issue de trois ateliers par l'ensemble des maires et les présidents des différents bureaux ASACO ; (iii) son mode de financement à travers les mairies, les partenaires financiers et les ASACO, épargnant ainsi aux ménages le paiement direct des coûts de transport des femmes enceintes (17).

\section{Les faiblesses}

Le financement de la caisse a connu d'énormes insuffisances, ainsi le taux de paiement des quotes-parts de 2006 à 2009, est de 43\% pour les mairies, $73 \%$ pour les ASACO et de $15 \%$ pour le conseil de cercle (18). Ce sous financement a conduit à la révision en 2013, aboutissant à un mécanisme de financement non avantageux pour les populations (paiement des quotes-parts 
par les ménages qui n’a pu atteindre que 12\%). Le financement de la caisse a manqué : (i) l'accompagnement des élus locaux par rapport aux engagements pris vis-à-vis de la caisse de solidarité ; (ii) une absence des réunions des comités de recouvrement et de celui de la surveillance de la caisse ; (iii) une sorte d'attentisme de l'apport de l'ONG MdM-Belgique. Cette attitude de la majeure partie des communes a découragé, en partie, les communes à jour de leur cotisation. A ceux-ci on pourrait ajouter le faible pouvoir économique de la population impactant sur le recouvrement des impôts au niveau des collectivités. Autres difficultés qui pourraient être citées comme faiblesses sont : $(7,18)$

o Le retard dans l'actualisation du partage des coûts des quotes-parts des années suivantes ;

o L'insuffisance d'information et de sensibilisation des communautés bénéficiaires ;

o La non-contribution des collectivités décentralisées au fonctionnement de la caisse, garant de la santé des communautés après la révision ;

o La perte progressive de « l'esprit de solidarité » autour de la référence par la non contribution de certains acteurs ;

o La contrainte géographique particulière de la zone ;

o L'absence de structure bancaire pour la sécurisation des fonds ;

o L'insuffisance dans la tenue des outils de collecte d'information ;

o L'absence d'accompagnement politique de la part des élus communaux et des ASACO.

Le financement de la caisse de solidarité du système référence/évacuation des femmes en difficulté d'accouchement, soulève la problématique des taux insuffisants de recouvrement. Cette situation pose d'énormes difficultés pour le fonctionnement du système en général. Les CSRéf se trouvant dans cette situation de dysfonctionnement s'endettent généralement auprès des fournisseurs de carburant (12). En dehors des difficultés du fonctionnement de l'ambulance, il y a aussi celui des groupes électrogènes dans les districts ruraux n'ayant pas d'électricité.

Encore, ce dysfonctionnement oblige les CSRéf à l'envoi des ambulances sans infirmiers ou sages-femmes et le perdiem du chauffeur est, de surcroit, à la charge de la famille de la parturiente. Ces mêmes difficultés ont été ressorties par Berthé, dans son étude sur les facteurs associés à la faible contribution des communautés dans le système de référence/évacuation dans le district de Bourem au Mali (18) ; par Traoré, dans son étude sur l'impact de la gratuité césarienne dans le CSRéf de Barouéli (22) et par Traoré, dans l'évaluation du système de référence/évacuation des urgences obstétricales dans le district sanitaire de la commune V à Bamako (19). La plupart des études font ressortir une insuffisance de l'accompagnement par les élus 
locaux. Dans l'étude de Traoré à Bamako, certains élus locaux ont décliné de répondre aux questionnaires (19).

\section{Conclusion}

La lutte contre la mortalité maternelle à travers le transport des urgences obstétricales ne sera possible que si la caisse de solidarité du système de référence-évacuation joue pleinement son rôle. Son importance pour l'atteinte des objectifs de la réduction des décès maternels et périnatals est reconnue et très apprécié au niveau des communautés rurales par l'ensemble des acteurs mais avec un taux de recouvrement très faible. L'obstacle majeur aujourd'hui pour la pérennisation de ce système réside dans l'organisation et l'engagement des collectivités décentralisées et des associations de santé communautaire pour améliorer leur financement. Ainsi, en tenant compte de la faible mobilisation des ressources au niveau des collectivités rurales, il parait indispensable de faire du plaidoyer pour promouvoir la santé par l'engagement communautaire.

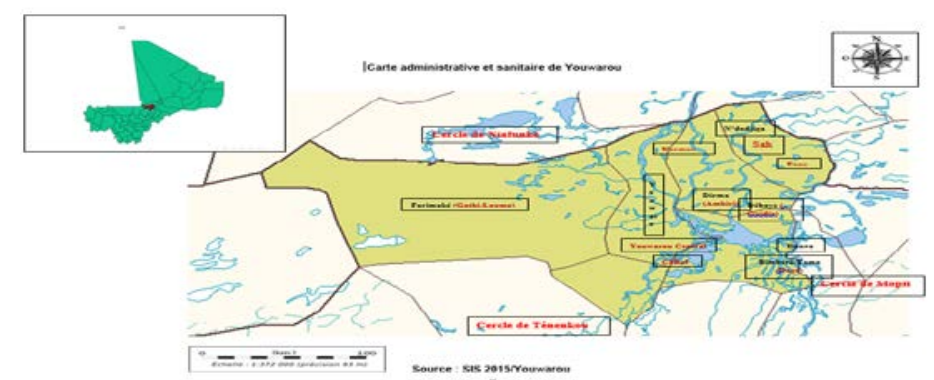

Figure 3. Carte administrative et sanitaire de Youwarou en république du Mali

\section{References:}

1. https://www.who.int/fr/news-room/fact-sheets/detail/maternalmortality consulté le 3/12/2020

2. Coulibaly, Salif, Fatoumata Dicko, Seydou Moussa Traoré, Ousmane Sidibé, Michka Seroussi et Bernard Barrère. 1996. Enquête Démographique et de Santé, Mali 1995-1996. Calverton, Maryland, USA : Cellule de Planification et de Statistique du Ministère de la Santé, Direction Nationale de la Statistique et de l'Informatique et Macro International Inc.

3. Maine D, Akalin MZ, Ward VM, Kamara A (1997). The design and evaluation of maternal mortality programs. Centre for Population and Family Health, School of Public Health, Columbia University, New York. 
4. De Brouwere V. Appui à la mise en œuvre et à l'évaluation du système de référence avec la périnatalité comme porte d'entrée dans le Cercle. UNICEF Mali. 1997 ;

5. Ministère de la santé et de l'hygiène publique Mali. Cadre conceptuel version finale. MSHP ; 2000.

6. Centre de Santé de Référence de Youwarou. Evaluation Référence Evacuation District Sanitaire de YouwarouCOR. Bamako ; 2016.

7. Ministère de la santé et de l'hygiène publique Mali. Evaluation externe du système de référence et évacuation Mali (005). Bamako : MSHP ; 2004.

8. Jahn A, De Brouwere V. La référence pendant la grossesse et l'accouchement : concepts et stratégies.

9. Walt G, Shiffman J, Schneider H, Murray S, Brugha R, Gilson L. "Doing" health policy analysis: Methodological and conceptual reflections and challenges.; In 2008. (Health Policy Plan; vol. 317).

10. Van Olmen J, Criel B, Van Damme W, Marchal B, Van Belle S, Van Dormael S. Analysing Health System Dynamics : A Framework. Stud Heal Serv Organ Policy. $2012 ; 2: 1-117$.

11. H L. A Comparative Approach to Policy Analysis. By H. M. Leichter. (Pp. 326; illustrated; £4.95.) Cambridge University Press: Cambridge. 1979.The Division in British Medicine. By F. Honigsbaum. (Pp. 445; illustrated; £6.95 pb.) Kogan Page: London. 1979. Psychol Med Cambridge University Press; 1981 Feb 9 ;11(1) :204.

12. Centre de Santé de Référence de Youwarou. Monitorage Réf-Eva ok. Bamako; 2010.

13. Ministère de la santé et de l'Hygiène publique du Mali. Guide pour la mise en œuvre de la gratuite de la césarienne. MSHP ; 2005.

14. Centre de Recherche en Reproduction Humaine et Démographie. Rapport de synthèse : l'évaluation de la politique de gratuité de la césarienne dans cinq zones sanitaires. Benin ; 2014.

15. ALLs BD et. Rapport final Césarienne. 2008.

16. Centre de Santé de Référence de Youwarou. Cadre Conceptuel de la Reference Evacuation de Youwarou. Bamako ; 2006.

17. Centre de Santé de Référence de Youwarou. Cadre Conceptuel Reference Evacuation Révisé Youwarou. Bamako ; 2013.

18. Berthé I. Facteurs associés à la faible contribution des communautés dans système de référence-évacuation dans le district sanitaire de Bourem (Mali) en 2010. [Ouidah] : IRESP ; 2011.

19. Traore A. Evaluation du système de Référence/Evacuation des urgences obstétricales dans le district Sanitaire de la Commune V en 2011. Bamako ; 2012. 
20. Boidin B, Laidet E, Manier R. La santé communautaire et ses défaillances dans la région de Kayes au Mali. Facts Rep. 2012;(8).

21. Sidibé F. Problématique de la Référence/ Evacuation au Centre de Santé de Yanfolila. [Bamako] : FMPOS ; 2004.

Traoré IS. Impact de la gratuite de la césarienne au Centre de Sante de Référence du cercle de Barouéli. [Bamako] : FMPOS ; 2007. 